\title{
Dynamic Foam: A nontechnical introduction to a novel hypothesis regarding discretized space-time and how gravity may be interpreted as information capacity
}

\author{
Joseph Geraci ${ }^{1}$ \\ ${ }^{1}$ Queen's University, Canada
}

January 5, 2020

\section{Abstract}

The ideas proposed here are based on a discretization of space-time inspired by theories such as Loop-Quantum Gravity [1] and corresponding follow up work [2]. There are some fundamental differences, the primary one being the inclusion of a novel degree of freedom that allows the space-time units to have some geometric flexibility, which we shall elaborate upon here. A natural consequence of our theory is that gravity can be interpreted as the information capacity of a region of space-time.

Idea: it has been proposed that a Planck space-time packet can encode a qubit of information, but what we propose is that the space-time volume of these packets can range from some minimum, to some maximum, asymptotically. The volume of the packet has no bearing on how much information is encoded: it is always one qubit. Here we propose that space-time consists of flexible discrete packets that are dynamic, and so we call these units Dynamic Foam or Dynamic Foam Packets (DFPs) when referring to the indivual units. The geometry of space-time in accordance with General Relativity emerges from this paradigm when you allow the DFPs to vary in volume according to how much gravity is present within a region of space-time.

This brief paper is meant to be an invitation to scrutinize this new idea before any major efforts are undertaken to use these ideas to construct a working quantum theory of gravity.

\section{Introduction}

The purpose of this little note is to introduce an idea about space-time for those interested in quantum gravity. This idea requires evaluation. The next paper to follow will be a more technical effort but here we will describe the idea and how the geometry of 
General Relativity can result naturally by adding a degree of freedom to the discretized units.

Essentially, we assume that a given volume of space-time remains fixed but that within this volume, space-time is made out of flexible discrete 4-D packets. The smallest possible units of space-time exist within regions where there are super dense masses, e.g., a black hole; next some base larger unit of space-time make up regions of space-time that are flat; and finally repulsive areas of space-time (if they exist), e.g., potentially the source of dark energy, will have units with larger volumes of space time. This last idea regarding repulsive space-time regions follows naturally from the fact that larger packets will cause a bulging of local space-time, but we do not postulate that this regime of space-time definitely exists.

\subsection{Essential Idea}

The idea can be summarized by the following hypotheses about space-time:

1. space-time is discretized

2. the 4-dimensional packets, i.e., the fundamental units of space-time, do not all have to be the same size

3. regions of space-time that are under the influence of high mass (high energy density), or where gravity has a powerful influence, is characterized by being made up of smaller packets

4. flat space-time have larger packets which may be considered the basic units of space-time

5. repulsive regions of space time are made up of DFPs with larger volumes, hence Dark Energy may be composed of DFPs that concentrate at the source and then shrink as one moves away from the phenomenon

6. Each dynamic foam packet or DFP, is capable of encoding one qubit of information

Natural consequences:

1. Black holes are capable of encoding the most information, the most massive being the most capable.

2. the space-time around a mass is naturally curved as the DFPs that tile a spacetime region will have differing volumes and will "tile" space-time with the smallest volumes at the center of the mass and gradually getting larger as we move farther from the mass. To imagine this, just look at a tiled floor and imagine what would happen to the floor if you suddenly increase the areas of the tiles in one region and gradually allow them to shrink. You would essentially warp your floor. 
3. Regions of space-time that are inversely warped, or which cause repulsion are possible and represent regions of space-time that have a lower capacity to encode information and may represent an aspect of dark energy.

4. There is a duality by which gravity can be interpreted: either as the curvature of space-time as we have in Einstein's theory, or the representation of how much information can be encoded in a region of space-time.

The DFP volumes can be modeled by a function that asymptotically approaches some lower limit for high gravity situations, and some upper limit for repulsive regions of space time.

\section{Overview of the theory}

A main consequence of this theory is that the amount of gravitational force within some volume of space-time is directly proportional to the amount of information that can be stored within that local space-time region. It is understood from previous work that the amount of information that can be encoded in space-time is maximal in black holes [3] as the density of matter is maximal, thus providing the highest number of micro-states in order to encode information. This, and the work from Loop Quantum Gravity (LQG), is a major influence for the perspective introduced in this paper. The ideas here are a simple addendum to the way we usually think of space-time in quantized theories of gravity, and a complete theory is not presented here.

A natural question that arises is, what is causing this dynamic variation in the DFPs? In the General Theory Of Relativity (GR) we have an interplay between the geometry of space-time and matter described by

$$
R_{\mu \nu}-\frac{1}{2} R g_{\mu \nu}+\Lambda g_{\mu \nu}=\frac{8 \pi G}{c^{4}} T_{\mu \nu}
$$

This beautiful relationship equates the geometry of space-time on the left with the mass/energy of an object via a set of ten coupled partial differential equations. In the DFP setting, the dynamics are played out via a mechanism that we don't provide here but the idea is that gravitons cause the variation in volume of the DFPs. In string theory, gravitons are predicted to exist, but here, some carrier particle is necessary for the theory to work at all. Here, the source of the mass or energy density requires some messenger to interact with the DFPs, so as to distort their local geometry, which will in turn be observable as the curvature described by the left hand side of equation (2).

One prejudice that has arisen among the public is that somehow the dimensional analysis that led to the idea of Planck units is sufficient to suggest that these values are fundamental and immutable. However, physicists have accepted that there could be smaller units of space time. We understand and accept that there could be issues with restricting space-time as having some ultimate fixed size, for example violations of the 
principle of Lorentz Symmetry, but we will not dive into this area. The LQG community is well aware of this issue as it arises naturally from the fundamental postulates of the theory.

Let's review the simple analysis that gives us the Planck units where $L_{P}, T_{P}$, and $E_{P}$ are the Planck length, time, and energy, respectively. For a reference please see [4].

$$
\begin{aligned}
& L_{P}=\sqrt{\frac{h G}{2 \pi c^{3}}} \\
& T_{P}=\sqrt{\frac{h G}{2 \pi c^{5}}} \\
& E_{P}=\sqrt{\frac{h c^{5}}{2 \pi G}}
\end{aligned}
$$

It is tempting to believe that these values represent certain fundamental aspects of the physics that govern our universe, and in a sense this is not opposed. These simple equations seem capable of revealing something fundamental that lies at the heart of massive gravitational phenomenon like black holes, and with a change in perspective, they tell us a story about how our reality is played out on some pixelated canvass.

As this relates to DFPs let's consider the following argument beginning with the Schwarzschild radius, that results from a solution to Einstein's equations. The Schwarzschild radius, $r_{S}$, of a mass is the radius at which the given mass in question would have to be shrunk to before it becomes a black hole. The relationship between a given mass and its corresponding $r_{S}$ is given by

$$
r_{S}=\frac{2 G M}{c^{2}}
$$

Now, recall that $E=M c^{2}$ and so one has

$$
r_{S}=\frac{2 G E}{c^{4}}
$$

Rearranging, one can say that in order to strictly contain this mass $M$ within a region $r_{S}$, or within some spatial uncertainty $\Delta x$, using more than

$$
E=\frac{c^{4}}{2 G} \Delta x
$$

energy, then a black hole would form. Thus we have that

$$
\Delta x=\frac{2 E G}{c^{4}}
$$

and

$$
\Delta x \geq \frac{E_{p} G}{c^{4}}=\sqrt{\frac{h c^{5}}{2 \pi G}} \cdot \frac{G}{c^{4}}=\sqrt{\frac{h g}{2 \pi c^{3}}}=L_{P}
$$


Thus, we have arrived at

$$
\Delta x \cdot \Delta t \geq \frac{L_{P}^{2}}{c}
$$

by noting that $\Delta x \geq L_{P}$ and that $\Delta x=\Delta t c$ and substituting. This uncertainty relationship suggests that distances below $L_{P}$ exist but that it comes with a price in terms of the precision one may have in the time dimension. It also suggests that spacetime is non-commutative beyond the $L_{P}$ limit.

The DFP framework suggests that the flexibility afforded by the uncertainty relationship derived, allows space-time to consist of packets of different sizes, and that gravity is a consequence of differing levels of density of space-time in terms of how many DFPs are packed into a given space-time volume. As each DFP carries an information storing capacity of 1 qubit, one can then see that flat regions of space-time represent baseline levels of information storage capacity, black holes the ultimate limit of storage capacity, and repulsive areas of space-time as having the lowest information storage capacity.

\section{A Toy Model}

Here we suggest that through the interaction of gravitons, that the DFP volumes may dynamically react to mass densities. We utilized a sigmoid ansatz to derive this toy model because we wanted to have certain asymptotic properties. For example, when a region of space-time is distant from any significant mass/energy density, then we wish space-time to be flat and be comprised of a mostly homogeneous set of DFPs with the same space-time volume. Here we assume that this volume consists of

$$
V_{P}=T_{P} \times L_{P}^{3}
$$

but this base unit may be smaller. In equation (2), note that $T_{\mu \nu}$ is referenced, which is the well known stress-energy tensor. This object encodes the energy density and flux present in some region of space time and is responsible for gravity in the theory of General Relativity. In our toy model we will refer to the energy density within a region of space-time as $\rho_{E}$, and one can think of this as the density of a mass in our simple picture. We do not concern ourselves with tensors at this stage of our exposition. The energy density affects the size of the DFPs depending on how far the source of it is.

A massive body with density equal to a less massive body will curve space-time more in the sense of equation (2). This means that in the DFP picture we want the ability to understand the dynamics at the Planck scale that gives rise to the relationship between energy and gravity as curvature. In DFP theory, the space-time packets closest to the center of mass of this massive body will deform to become smaller, while the overall volume of space-time of this region remains constant. Therefore, this local shrinking of the DFPs will allow there to be more of them, and thus the density of space-time itself will increase, and finally, this leads to a higher information storing capacity. This naturally causes the curvature described by (2). The heart of this toy model is given by 


$$
V\left(d, \rho_{E}\right)=\frac{V_{P}}{1+\beta \exp \left(\frac{-d^{2}}{\rho_{E}} \frac{\rho_{o}}{d_{o}}\right)}
$$

where $V$ is the volume of a given DFP, $d$ is the distance from the source to the DFP in question, $V_{P}$ is from equation $(12)$ and has units of space-time volume, $\rho_{E}$ is the energy density of the object in question (and extracted from $T_{\mu \nu}$ ). $\rho_{o}$ and $d_{o}$, are reference density and distance values to remove units so that equation (13) has units of space-time volume. Specifically the given volume of a DFP depends on its distance $d$ to an object of energy density $\rho_{E}$. $\beta$ is a parameter which determines how small the DFPs can get under high gravitational situations. Note that in the limit

$$
\begin{aligned}
d \rightarrow \infty & \Longrightarrow V \rightarrow V_{P} \\
d \rightarrow 0 & \Longrightarrow V \rightarrow \frac{V_{P}}{1+\beta} \\
\rho_{E} \rightarrow \infty & \Longrightarrow V \rightarrow \frac{V_{P}}{1+\beta} \\
\rho_{E} \rightarrow 0 & \Longrightarrow V \rightarrow V_{P}
\end{aligned}
$$

which is exactly what we require. This means that in the limit where we have $T_{\mu \nu}=0$ space-time should be flat and thus the DFPs should have volume at the base value, which we have assumed was $V_{P}$, but may not be. However, when there is some energy density to speak of, then the the DFP volumes can shrink near to $\frac{V_{P}}{1+\beta}$. The distance from the mass/energy source to the DFPs will be the factor that determines the differing sizes of the packets, and thus the curvature that results. A next step would be to work out precisely the relationships between the DFPs and the left hand side of equation (2). This effort will be saved for future work, it this approach merits it. A more complete approach towards a quantum theory of gravity can then be sought where the graviton interaction with the DFPs will be responsible for this local change in geometry and for the local information storage capacity of regions of space-time. This toy model however suggests something fundamental about this picture of spacetime: it would behave like a fluid consisting of constituent particulates of differing sizes, potentially with statistical mixtures, where high gravitational situations will cause the smaller DFPs to pack together.

\section{Bekenstein-Hawking Entropy}

From the perspective of DFPs, black holes occupy the highest possible number of packets, with the larger proportion of them hiding deep within. Though there is no longer a singularity from our perspective, there is some ultimate density of DFPs that now replaces the singularity. In this sense black holes are capable of storing more information than any other object. From the perspective of Landauer's Principle [5], the erasure of 
a bit of information costs energy. Specifically, one bit of information being erased from a system in a heat bath of temperature $\mathrm{T}$ (in Kelvins) will create

$$
Q=k T \ln 2
$$

heat, where $k$ is the Boltzmann constant. Smaller black holes are capable of losing mass as they become sufficiently warm. In the DFP picture, as a black hole shrinks through Hawking radiation, it means that it must occupy fewer DFPs. This means that information storage capacity is being returned to the universe, and thus its entropy has increased. Now, assuming that it, in the process of shrinking, gives up $N_{D F P s}$ packets of space-time, and thus as many qubits, we can compute

$$
Q=k T \ln 2 \cdot N_{D F P s}
$$

as the heat generated by this erasure process within a black hole. The black hole is sitting in a $2.7 \mathrm{~K}$ heat bath, radiating Hawking radiation, and thus this interpretation may be something worth considering.

As a final theoretical consideration consider the Bekenstein-Hawking radiation formula [3] given by

$$
S_{B H}=\frac{A}{4 L_{P}^{2}}
$$

From the DFP perspective, this would be corrected as the Planck length is shorter within a black hole and actually varies from being the smallest nearest to the source (classically what would be referred to as the singularity) while getting larger as one approaches the event horizon and beyond. Thus, the DFP approach estimates a higher entropy in terms of the number of micro-states that the black hole surface occupies.

\section{Conclusion And The Potential For Testing The DFP Hypothesis}

We propose a new degree of freedom for discrete space-time units. In Loop Quantum Gravity they are assumed to have dimensions $T_{P}$ and $L_{P}$, and here we propose that they shrink near sources that cause large gravitational effects such as large concentrated masses. They then expand to some base size for flat space (we used $T_{P} \times L_{P}^{3}$ but this is just an assumption), i.e., when $T_{\mu \nu}=0$, and can potentially grow beyond this size which would create repulsive areas of space-time. This causes a natural tiling and warping that can be made to accord with the geometry of the General Theory Of Relativity. This picture immediately implies that those areas of space time that are warped due to the presence of some energy density, are capable of encoding more information than flat or repulsive regions of space-time. This is due to the correspondence of each DFP being able to encode one qubit of information. High gravity situations cause smaller packets to form, and thus these regions will have more DFPs to pack together, as volume is conserved. Thus, we are aligned with other methods that assume a minimum Plancklike distance. 
How can we experimentally evaluate the DFP proposal? Fortunately, experiments have already been conducted that is testing constraints on Lorentz invariance with attempts at observing the discrete structure of space time. Two such efforts are given by [6] [7] where the first is utilizing observations from a distant large energy gamma radiation source, and the latter proposes an experiment that next generation Compton and pair-production telescopes may be able to accomplish. This means that testing the discrete structure of space-time between high gravity areas vs flatter areas, may be able to detect differences, and thus accord with DFP theory. Discrepancies in the sizes of the discreteness of space-time measured would align with the tenets of DFP theory. This would be a costly endeavor as one might have to mount an apparatus on a satellite to perform experiments farther from Earth for a comparison. There may be other ways around this but further consideration will be required. For now, the DFP proposal is just another perspective that may lead to a novel theory of quantum gravity. After some scrutiny, the next effort will be to actually work out a quantization scheme and work out the representations for the DFPs. Other consideration will have to be made, specifically the statistical nature of the dynamic foam structures. Quantum entanglement will need to be incorporated between the packets in addition to quantum information theoretic aspects of the theory. In this way, there may be hope for a theory where the paradigm of quantum field theory suggests a mechanism for the interaction of gravitons with space-time itself to produce a geometric effect that, in the classical limit, agrees with the General Theory Of Relativity.

\section{References}

[1] L. Smolin. An invitation to loop quantum gravity. https://arxiv.org/pdf/hepth/0408048.pdf, 2008.

[2] Alejandro Perez. Black holes in loop quantum gravity. Reports on Progress in Physics, 2017. doi: 10.1088/1361-6633/aa7e14.

[3] Jacob D. Bekenstein. A Universal Upper Bound on the Entropy to Energy Ratio for Bounded Systems. Phys. Rev., D23:287, 1981. doi: 10.1103/PhysRevD.23.287.

[4] D. Meschini. Planck-scale physics: Facts and beliefs. Foundations of Science, Springer, 12:277-294, 2006. doi: 10.1007/s10699-006-9102-3.

[5] C. Fuchs. Landauer's principle and black-hole entropy. In Workshop on Physics and Computation, pages 86-92, Oct 1992.

[6] P. Laurent, D. Götz, P. Binétruy, S. Covino, and A. Fernandez-Soto. Constraints on lorentz invariance violation using integral/ibis observations of grb041219a. Phys. Rev. D, 83:121301, Jun 2011.

[7] Fabian Kislat and Henric Krawczynski. Planck-scale constraints on anisotropic lorentz and cpt invariance violations from optical polarization measurements. Phys. Rev. D, 95:083013, Apr 2017. 